\title{
High Resolution Image Reconstruction from Projection of Low Resolution images differing in Subpixel shifts
}

\author{
Manohar Mareboyana ${ }^{1}$, Jacqueline Le Moigne ${ }^{2}$, Jerome Bennett ${ }^{3}$
}

\begin{abstract}
In this paper, we demonstrate a simple algorithm that projects low resolution (LR) images differing in subpixel shifts on a high resolution (HR) also called super resolution (SR) grid. The algorithm is very effective in accuracy as well as time efficiency. A number of spatial interpolation techniques using nearest neighbor, inverse-distance weighted averages, Radial Basis Functions (RBF) etc. used in projection yield comparable results. For best accuracy of reconstructing SR image by a factor of two requires four LR images differing in four independent subpixel shifts. The algorithm has two steps: i) registration of low resolution images and (ii) shifting the low resolution images to align with reference image and projecting them on high resolution grid based on the shifts of each low resolution image using different interpolation techniques. Experiments are conducted by simulating low resolution images by subpixel shifts and subsampling of original high resolution image and the reconstructing the high resolution images from the simulated low resolution images. The results of accuracy of reconstruction are compared by using mean squared error measure between original high resolution image and reconstructed image. The algorithm was tested on remote sensing images and found to outperform previously proposed techniques such as Iterative Back Projection algorithm (IBP), Maximum Likelihood (ML), and Maximum a posterior (MAP) algorithms. The algorithm is robust and is not overly sensitive to the registration inaccuracies.
\end{abstract}

Keyword: Super Resolution, High spatial resolution, Remote sensing Data

\section{INTRODUCTION}

Spatial resolution of remotely sensed images plays an important role in analysis and visual interpretations. Spatial resolution corresponds to the size of the pixel on the ground. Smaller the ground area represented by a pixel is larger is the spatial resolution and gives more detail about the observation. Unfortunately the spatial resolution of remote sensing system cannot be increased without limit. The limiting factors are diffraction limits, noise, and spacecraft orbits. An excellent description of limiting factors of ground resolution by optical systems onboard satellites is given in [1].

Super resolution (SR) is the process of artificially increasing the spatial resolution of an image corresponding to a scene given a set of observed low resolution (LR) images of the same scene that differ in sub-pixel translation. In this paper we describe different techniques constructing high resolution images super resolution of improving the ground resolution from multiple images of the same area on the ground but with subpixel shifts. . The low resolution images of the scene provide complementary information that will be used to estimate pixel values in denser grid. Constructing a SR

\footnotetext{
${ }^{1}$ Bowie State University, mmareboyana@bowiestate.edu

2,3 Goddard Space Flight Center, Greenbelt, MD
} 
image from a set of LR images is basically a constrained ill-posed problem, because there is no unique solution. The interest in this area of research is spurred by the seminal paper by Tsai and Huang [2].

There are a number of approaches to constructing SR image from a set of LR images. An excellent review of the techniques used is given in [2]. They can be divided broadly into a) Frequency Domain b) Spatial Domain approaches using interpolation and statistical methods, c) wavelet based, and d) set theoretic methods.

\section{SPATIAL DOMAIN APPROACHES TO SR}

A variety of techniques exist in spatial domain approaches among which interpolation and regularization, statistical methods, and projection onto convex sets. The advantages of spatial domain approaches are that unconstrained motion between frames can be handled easily and a-priori knowledge can be incorporated in regularization.

\subsection{Interpolation}

Consider estimating SR image $\mathrm{X}$ from multiple $\mathrm{LR}$ images $\mathrm{Y}_{1}, \mathrm{Y}_{2}, . . \mathrm{YK}$. $\mathrm{Y}_{\mathrm{k}}$ is related to $\mathrm{X}$ as

$$
\mathrm{Y}_{\mathrm{k}}=\mathrm{D} \mathrm{F}_{\mathrm{k}} \mathrm{B} \mathrm{X}+\mathrm{N}
$$

Where $X$ and $Y_{k}$ are HR and let LR images represented as vectors with lexicographically ordered pixels, $B$ is spaceinvariant blurring kernel, $\mathrm{F}_{\mathrm{k}}$ is motion operator, $\mathrm{D}$ is down sampling operator, and $\mathrm{N}$ is noise term . Combining all three operators into a single operator $\mathrm{H}_{\mathrm{k}}$ for kth frame, the above relation can be given as follows.

$$
\mathrm{Y}_{\mathrm{k}}=\mathrm{H}_{\mathrm{k}} \mathrm{X}+\mathrm{N}
$$

Where $\mathrm{H}=\left[\mathrm{H}_{1}, \mathrm{H}_{2}, . . \mathrm{H}_{\mathrm{k}}\right]^{t}$ and incorporates, blurring, motion and down sampling of SR image X. Considering blurring and down sampling are space-invariant, $\mathrm{X}$ can be estimated from motion operator given $\mathrm{Y}$. The noise component makes the solution to this inverse problem even more difficult. Thus the interpolation techniques rely on motion operator and regularization of interpolated images to account for noise. An approach to estimating SR image using interpolation techniques is shown in a block diagram.

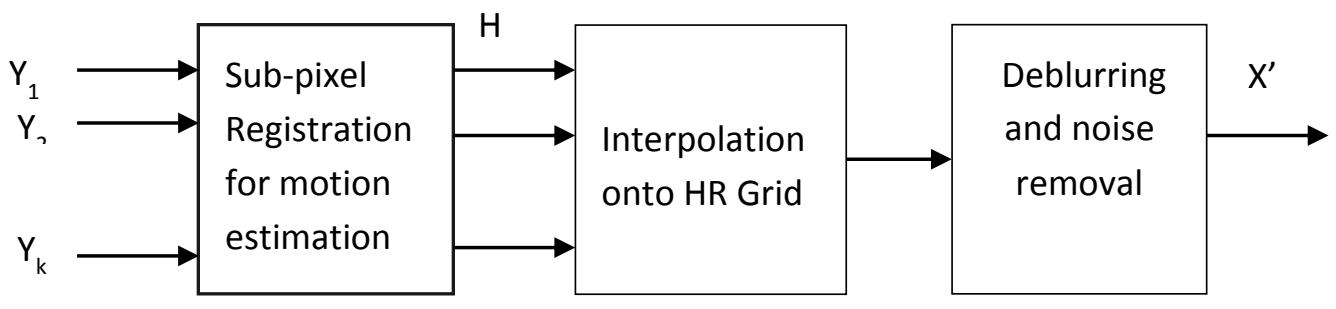

Figure 1. Block diagram reconstructing HR image from LR images

The motion parameters $(\mathrm{H})$ are estimated by using sub-pixel accuracy image registration techniques [3-4]. One of the images from LR $\left(\mathrm{Y}_{1}\right)$ is used as reference image and the motion (translations) are computed to sub-pixel accuracies $(\mathrm{H})$. When all the SR images $\left(\mathrm{H}_{\mathrm{k}} \mathrm{Y}_{\mathrm{k}}\right)$ are overlaid, a composite image of non-uniformly spaced pixels is obtained. Non- 
uniform interpolations are applied to estimate pixel values on HR grid. The interpolated image is restored by removing the blur (due to PSF) and noise. The non-uniform interpolation techniques are both direct and iterative.

i. Nearest Neighbor: The simplest interpolation used in constructing a HR image from a set of LR images is to assign the closest LR image pixel value to every point in HR grid. Obviously, it is the fastest interpolation and uses a single LR-image for a given HR grid point [5].

ii. Bi-Linear/Bi-Cubic Interpolation: Bi-Linear interpolation assumes piece-wise linear model. For every HR grid point the closest LR image point along with its three neighbors are used in bi-linear interpolation. This method like nearest neighbor interpolation uses a single LR image for estimating the pixel value at a given point in HR grid. A more accurate method uses bi-cubic interpolation from LR image closest to the HR grid point.

iii. Interpolation from Scattered Points: Image interpolation on uniformly gridded data is well known and a number of techniques linear, cubic, and spline interpolations [6-7] exist in literature. In the context of super resolution, the interpolation of scatter of points (non-uniformly gridded points) is applicable.

a) Scattered interpolation: Given a set of $\mathrm{k}$ values in 3 -D space points $(\langle\mathrm{x} 1, \mathrm{y} 1, \mathrm{v} 1\rangle,\langle\mathrm{x} 2, \mathrm{y} 2, \mathrm{v} 2\rangle, . .\langle\mathrm{xk}, \mathrm{yk}$, $\mathrm{vk}>$ ), one can construct a surface of the form $\mathrm{F}(\mathrm{x}, \mathrm{y})$ passing through all the given points. The value $\mathrm{v}=$ $\mathrm{F}(\mathrm{xq}, \mathrm{yq})$ at any query point $(\mathrm{xq}, \mathrm{yq})$ is the scatter interpolation.

In the case of linear interpolation $\mathrm{F}$ is vector consisting of three coefficients of a plane $(\mathrm{v}=\mathrm{ax}+\mathrm{by}+\mathrm{c})$ at point $(\mathrm{x}, \mathrm{y})$.

$\mathrm{V}=\mathrm{F}^{\mathrm{t}} \mathrm{X}_{\mathrm{q}}$

where $\mathrm{X}_{\mathrm{q}}=[\mathrm{xq}, \mathrm{yq}, 1]^{\mathrm{t}}$ and $\mathrm{F}$ is given by

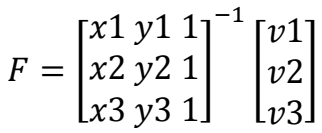

While any of the three points out of $k$ known points can be used, the three closest points (in Euclidean distance) will yield better interpolation result.

Better interpolation techniques can be applied by fitting a surface to $k$ known points such as cubic surface, cubic spline, or a polynomial of $k$ - 1 degree. Lagrangian interpolation in multi-dimension [8] is of special interest because of computational simplicity.

\subsection{Statistical Approaches}

In statistical approaches, the SR image is reconstructed from a set of LR. The observed LR image (y1, y2,.. yk) are treated as random variables and using these data, and prior information An optimal HR image ( $\mathrm{x}^{\prime}$ ) is estimated. A straightforward method involves maximizing the posterior $\mathrm{P}(\mathrm{x} \mid \mathrm{y} 1, \mathrm{y} 2, \ldots \mathrm{yk})$ from observed LR images. This method called maximum a posterior (MAP) is given by $\mathrm{x}=\operatorname{argmax}(\mathrm{p}(\mathrm{x} \mid \mathrm{y} 1, \mathrm{y} 2 . . \mathrm{yk})$ 
where $\mathrm{p}(\mathrm{x} \mid \mathrm{y} 1, \mathrm{y} 2 . . \mathrm{yk})$ is the posterior and is given by

$$
\mathrm{p}(\mathrm{x} \mid \mathrm{y} 1, \mathrm{y} 2 . . \mathrm{yk})=\frac{p(y 1, y 2, . . y k \mid x) p(x)}{\int_{x} \mathrm{p}(\mathrm{y} 1, \mathrm{y} 2 . . \mathrm{yk} \mid \mathrm{x}) p(x)}
$$

The numerator of $\mathrm{p}(\mathrm{x} \mid \mathrm{y} 1, \mathrm{y} 2 . . \mathrm{yk})$ is called likelihood function. The $\mathrm{x}$ that maximizes the likelihood function is called maximum likelihood estimate (MLE).

Considering the $\mathrm{y} 1, \mathrm{y} 2 . . \mathrm{yk}$ are statistically independent and

$$
p\left(y_{i} \mid x\right)=\frac{1}{\sqrt{2 \pi \sigma^{2}}} e^{-\left(y_{i-H_{i}} x\right)}
$$

$\mathrm{H}_{\mathrm{i}}$ operator combines down sample of HR image $\mathrm{x}$, and translation parameters.

$p\left(y_{1}, y_{2}, \ldots y_{k} \mid x\right)=\Pi_{i=1}^{k} p\left(y_{i} \mid x\right)$

MLE estimate of the $\mathrm{x}$ can be obtained by differentiation log likelihood function with respect to $\mathrm{x}$ and equating to zero. The optimal estimate thus turns out to be $\mathrm{x}=\frac{1}{k}\left(\sum H_{i}^{-1}\left(y_{i}\right)\right)$

\subsection{Iterative Back Projection (IBP)}

In IBP method, the initial HR image is reconstructed from the LR low resolution image by replicating pixels. The initial estimate is projected on the LR images by using the registration parameters and compared with observed LR images.

The difference is used to correct the HR image. The process is iterated until convergence. The algorithm is described in [9]. The algorithm is slower compared to non-iterative methods.

\section{THE GENERATIVE MODEL OF LR IMAGES}

For experimental purposes, the HR image(x) is used to generate LR images (y1,y2, .. yk). The generative model is as given below in Figure 2.

$$
y_{i}=T_{i} B D(x)+n_{i}
$$

$\mathrm{T}_{\mathrm{i}}, \mathrm{C}, \mathrm{D}$ are warping (translation only for $\mathrm{y}_{\mathrm{i}}$ ), blurring and down sample functions. We use blurring as unit function and zero additive noise in our experiments.

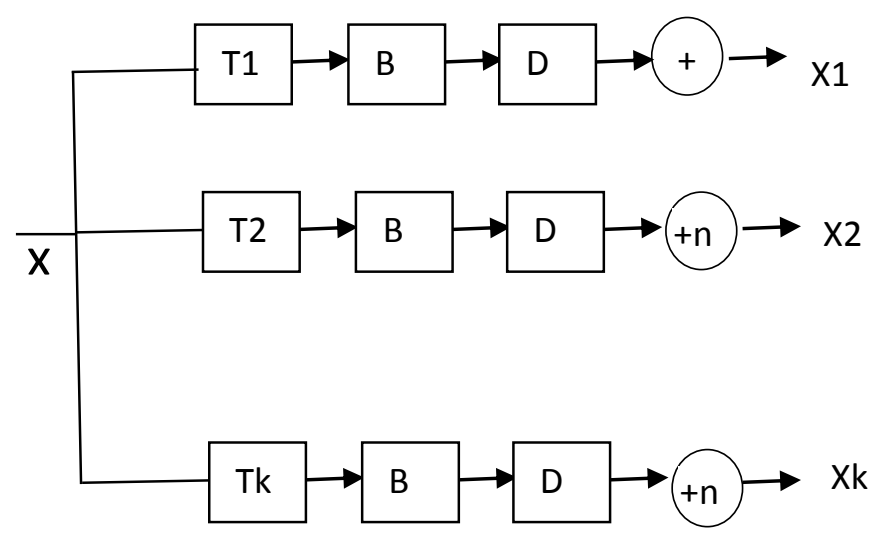

Figure 2: Generative model of LR images 


\section{INTERPOLATION BASED ALGORITHMS}

In the reconstruction of HR image by a factor of 2 , one needs 4 LR images differing in subpixel shifts. To reconstruct perfect HR image, the shifts should coincide with the missing pixels from the HR grid as shown in the Figure 3.

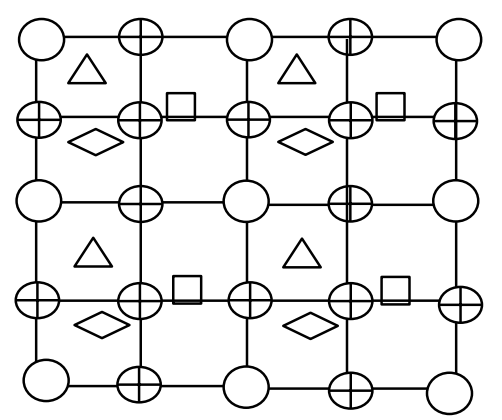

$\bigcirc$ LR Reference on HR

$\triangle \square \diamond$ LR Images with subpixel shifts

$\bigoplus$ Interpolated image on HR grid

Figure 3: The LR images super imposed on HR

\subsection{Overlaying LR images on HR grid based on NN Interpolation}

We consider reconstructing HR image(x) by factor of 2 from four LR images (y1,y2,y3,y4) differing in subpixel shifts as show in Fig. 3. The algorithm finds the closest LR from the missing pixels from HR grid and overlays the LR images $A=\pi r^{2}$ on HR grid by shifting it to the missing pixel position from HR grid. Considering y1 as the reference LR image and overlays on every other cell of HR grid along the row and column as shown in figure by circles. The other LR images are shown in triangles, square and diamond symbols.

$$
x^{\prime}=U^{2}(x 1)+\sum_{k=2}^{4} U^{2}\left(T_{k}\left(y_{k}\right)\right.
$$

In the above equation, $\mathrm{U} 2$ is the up sample by 2 operator and Tk is the shift to the missing pixel position of the HR grid of the nearest neighbor of the LR image.

\subsection{Inverse Distance Weighted (IDW) Interpolation}

Unlike in NN, in IDW all the LR images contribute to the estimation of missing pixels of HR grid based on the distance of the LR image pixels from the missing HR grid point. Initially the reference image fills up every other HR grid point in every row and column and the missing points are estimated from remaining LR images using inverse distance weightages.

$$
x^{\prime}=\sum_{k=2}^{4} w_{k} y_{k} \text { where } \sum_{k=2}^{4} w_{k}=1
$$




\subsection{Interpolation Using Radial Basis Functions (RBF)}

A radial basis function (RBF) is a real valued function whose value depends on the distance from a given point $\mathrm{x}_{\mathrm{i}}(\mathrm{x}$ a variable not to be confused with image variable used for HR image).

$$
\emptyset\left(x, x_{i}\right)=\emptyset\left(\left\|x-x_{i}\right\|\right)
$$

When the distance function is Gaussian, the RBF is called Gaussian RBF.

$$
\emptyset\left(x, x_{i}\right)=e^{-\left(x-x_{i}\right)^{2}}
$$

The reference LR image is used to fill up every other HR grid pixels along the row and column. The remaining LR images are used to estimate the 3 missing cells of $2 \times 2$ HR grid as follows

$$
x(i)=\sum_{k=2}^{4} \emptyset(i-T(k)) * y_{k}
$$

In the above equation vector $\mathbf{i}$ is the position of the missing pixel from HR grid $(0,1$ for horizontal, 1,0 for vertical and 1,1 for diagonal) $\mathrm{T}(\mathrm{k})$ is the distance of $\mathbf{i}$ from LR image $\mathrm{y}^{\mathrm{k}}$.

\section{EXPERIMENTAL RESULTS USING REMOTE SENSING IMAGES}

Figure 4(a) shows the Landsat image of the Chesapeake Bay. In Figure 4(b), the LR images shifted by using random subpixel shifts along width and height are shown. The LR images are generated from the image given in Fig. 4(a) using the generative model given in Section 3. The LR image is considered to be the reference image. The other images are subpixel shift version of the reference image. The shifts are randomly generated and the images are translated using these shifts. While constructing the HR image from the LR images, we do not use the shifts that were used in the generation of these images. We compute the shifts by using registration algorithm developed by Le-Moig,ne., et al [34].

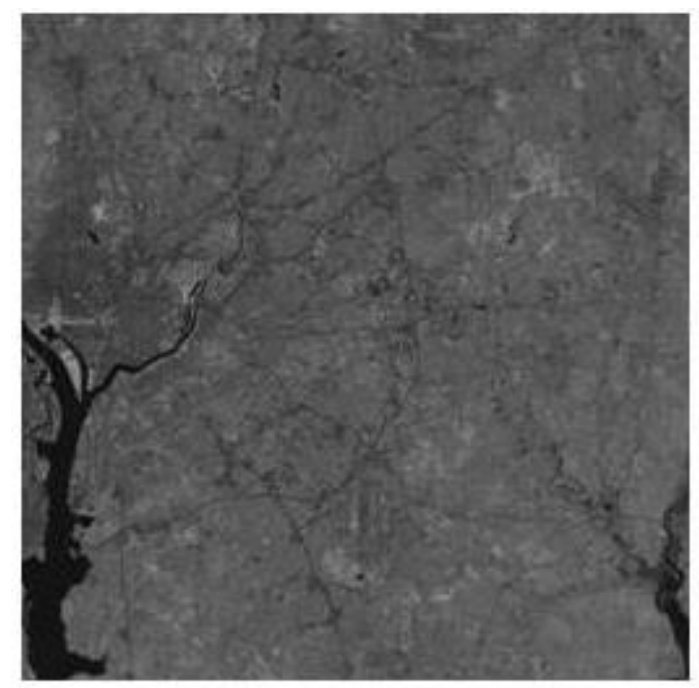

Figure 4(a): Chesapeake Bay Image 256x256 

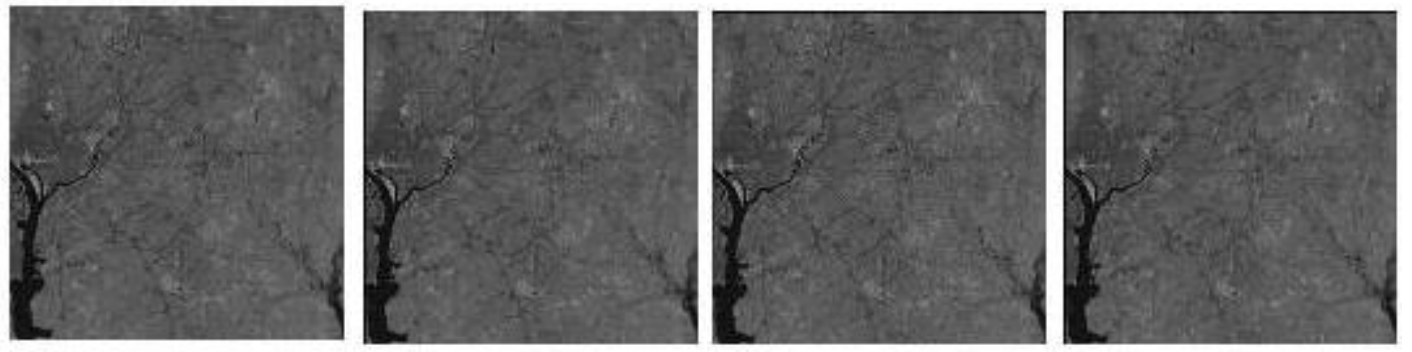

Figure 4(b): Simulated images 128x128 using random shifts along width and height

The first image of Figure 4(b) is used as the reference image and the remaining images shifted using random shift parameters.

The translational parameters of the shifted images are determined by using registration algorithm described in [3-4]. The algorithm based on wavelet decomposition is able to register given image with reference image to subpixel accuracies. The registration accuracies obtained by the algorithm are quite accurate. The difference between actual shifts vs. those computed by the registration algorithm given in [3-4] is less than $1 \%$.

We have tested reconstruction of HR image from the images given in Fig. 4(b) and compared the reconstructed image with original image given in Fig. $4 \mathrm{a}$ in terms of sum of squared error (SSE).

The Table 1 compares the accuracy of reconstruction of HR image ( ${ }^{\prime}$ ) from given LR images (y1,y2,y3,y4).

Translation parameters obtained for $\mathrm{y} 2, \mathrm{y} 3$, and $\mathrm{y} 4$ with respect to $\mathrm{y} 1$ are computed using the registration software given in [3-4]. The translational computed for $\mathrm{y} 2, \mathrm{y} 3, \mathrm{y} 4$ with respect to $\mathrm{y} 1$ are given below (tr is row shift, tc is column shift)

$\mathrm{y} 2: \operatorname{tr} 2=0.9, \mathrm{tc} 2=0.24$

$\mathrm{y} 3: \operatorname{tr} 3=0.12, \mathrm{tc} 3=0.82$

y4: $\operatorname{tr} 4=0.9, \operatorname{tc} 4=0.55$

The sum of squared errors (SSE) between the original HR image (x) and reconstructed HR image ( $\mathrm{x}$ ') using different methods are presented in the Table 1.

\section{TABLE 1}

\begin{tabular}{|l|l|}
\hline Algorithm & SSE \\
\hline NN(Nearest Neighbor) interpolation & 87799 \\
\hline IDW (inverse Distance Weighted) & 128624 \\
\hline MLE (Maximum Likelihood) & 167781 \\
\hline IBP (Iterative Back Projection) & 220468 \\
\hline RBF (Radial Basis Function) Interpolation & 77732 \\
\hline
\end{tabular}

In Table 2, the performance of the same algorithm for translation parameters shown below. 
$\mathrm{y} 2: \operatorname{tr} 2=082, \mathrm{tc} 2=0.08$

$\mathrm{y} 3: \operatorname{tr} 3=0.17, \mathrm{tc} 3=0.84$

$\mathrm{y} 4: \operatorname{tr} 4=0 . .64, \operatorname{tc} 4=0.56$

Table 2:

\begin{tabular}{|l|l|}
\hline Algorithm & SSE \\
\hline NN(Nearest Neighbor) interpolation & 886717 \\
\hline IDW (inverse Distance Weighted) & 120953 \\
\hline MLE (Maximum Likelihood) & 167938 \\
\hline IBP (Iterative Back Projection) & 226565 \\
\hline RBF (Radial Basis Function) Interpolation & 71946 \\
\hline
\end{tabular}

In these experiments, the RBF and NN consistently performed better than other algorithms. The algorithms are very sensitive to the row shift and column shifts. Perfect reconstruction is of course possible if the shifts are such the missing pixels are presented in $\mathrm{y} 2, \mathrm{y} 3$, and $\mathrm{y} 4$.

\section{CONCLUDING REMARKS}

Various interpolation algorithms are implemented to compare their performance in reconstructing HR image from a set of LR images differing subpixel shifts. Interpolation using RBFs performed the best in most cases. HR reconstruction accuracy depends on the subpixel shifts and number of LR images. Increasing the spatial resolution by a factor of two requires 4 LR images with different subpixel shifts. Increasing the HR spatial resolution by a factor of $n$ of course requires $\mathrm{n}^{2} \mathrm{LR}$ images with different (non-repeating) subpixel shifts. In this paper, we have experimented with four LR images to increase the spatial resolution by a factor of 2 . Further research is required to improve the spatial resolution by an arbitrary number with a given number of LR images.

\section{REFERENCES}

1. Chesley, B., Lutz, R, and Brodsky, R., Space Payload Design and Sizing (Chapter 9, pp. 255-272) in book by Wetz J.R and Larson, W.J., Space Mission Analysis and Design (Third Edition), published by Microcosm Press and Kluwer Academic Publishers, 1999.

2. Yang, J., and Huang, T., Image super-resolution: Historical overview and future challenges, Google book library 2013.

3. Le-Moig,ne, J.; Wei Xia; Chalenriwat, P.; EI-Ghazawi, T.; Mareboyana, M.; Netanyahu, N.; Tilton, J.C.; Campbell, W.J.; Cromp, R.P., "First evaluation of automatic image registration methods," Geoscience and Remote Sensing Symposium Proceedings, 1998. IGARSS '98. 1998 IEEE International, vol.1, no., pp.315,317 vol.1, 6-10 Jul 1998.

4. Le-Moigne, J., Nethanyahu, N. S., Eastman, R. D., Image Registration for Remote Sensing, Cambridge University Press, 2011.

5. Nguyen, N., Milanfar, P. and Golub, G, “A computationally efficient superresolution image reconstruction algorithm," IEEETransactions on Image Processing, vol. 10, pp. 573-583, Apr 2001.

6. Keys, R. G., Cubic Convolution Interpolation for Digital Image Processing, IEEE Transactions on Acoustics, Speech, and Signal Processing, Vol. ASSP-29, No. 6, December 1981, pp.1153-1160 
7. Hou, H. S., and Andrews, H. C., Cubic Splines for Image Interpolation and Digital Filtering, , IEEE Transactions on Acoustics, Speech, and Signal Processing, Vol. ASSP-26, No. 6, December 1978, pp.508-517

8. Lehmann, T. M., Gonner, C. and Spitzer, K., Survey: Interpolation Methods in Medical Image Processing, IEEE Transactions on Medical Imaging, Vol. 18, No. 11, November 1999.

9. Irani, M and Peleg, S., Improving resolution by Image Registration, CVGIP, Graphical Models and Image Processing, Vol. 53, No.3, May 1991. 